\title{
Numerical modeling of the haemodynamics of a left ventricular assist device (LVAD) prototype
}

\author{
F. Lagasco ${ }^{1}$, M. Castelanelli ${ }^{1}$, G. Vezzani ${ }^{1}, \&$ M. Ercolani ${ }^{2}$ \\ ${ }^{1} D^{\prime}$ Appolonia S.p.A., Genoa, Italy \\ ${ }^{2}$ Tecnobiomedica S.p.A., Pomezia, Italy
}

\begin{abstract}
One of the main concerns when designing Left Ventricular Assist Devices (LVADs) is ensuring minimisation of thrombosis potential. In fact thrombus formation and resulting thromboembolism, which depend on the haemocompatibility of contact surfaces and fluid dynamics of the blood flow, are major risks associated to the use of LVADs as a long-term or permanent implantation. In the present study an existing LVAD prototype is numerically modeled with the aim to investigate the fluid motion field during the whole cardiac cycle of ejection and filling. 3D predictive numerical results are discussed against available experimental results.
\end{abstract}

\section{Introduction}

Among the causes inducing thrombus formation are the fluid dynamics of the blood flow, which are in turn strongly dependent on the device geometry. Severe blood activation leading to thrombosis is attributed to a number of adverse flow characteristics including turbulence, recirculation and stasis. High levels of turbulence intensity and boundary shear, in conjunction with long exposure times are also undesirable since resulting shear stresses can induce lysis.

The present work is aimed to predict the performance of the LVAD prototype developed by Tecnobiomedica (Italy) with respect to the flow characteristics experienced throughout a complete cardiac cycle. The motion is numerically modeled by means of a commercial CFD code (FIDAP), which employs a finite element method to solve the governing equations of the three-dimensional, unsteady flow of an incompressible Newtonian fluid. In order to achieve a 


\section{Simulations in Biomedicine $V$}

sensitive judgement on the numerical results prior to comparing with experimental evidence, two models with different levels of accuracy have been developed.

\section{Prototype characteristics}

LVAD is designed to receive blood from the apex of the left ventricle of the natural heart and to eject blood into the aorta. Devices can be implanted in the human body or placed outside the body with connections through the chest wall. The Tecnobiomedica implantable device under analysis consists of a driven pusher plate activated by a microprocessor based control system and acting on a poliurethane cardiac chamber membrane (Figure 1). Inlet and outlet ports connect the left ventricle and the aorta by means of flexible conduits (cannulae). Each port is fitted with a tilting disk valve (Sorin Biomedica Cardio, Italy): the inlet valve is oriented to project the flow toward the inside chamber wall whilst the outlet valve is oriented to take advantage of the rotational washing motion existing at late filling. The polyurethane chamber, $0,35 \mathrm{~mm}$ thick, presents a maximum diameter of $84 \mathrm{~mm}$, which equals the diameter of the pusher plate, and a maximum height of $20.4 \mathrm{~mm}$. Inlet and outlet tubes are $21.4 \mathrm{~mm}$ in diameter. The cardiac natural function is replaced by the periodic movement of the pusher plate: when it travels downwards, blood is ejected through the outflow tube while the inflow tube is closed (ejection phase). At the end of this phase, the piston has covered a stroke length of $17 \mathrm{~mm}$, leaving a clearance of around $3 \mathrm{~mm}$.

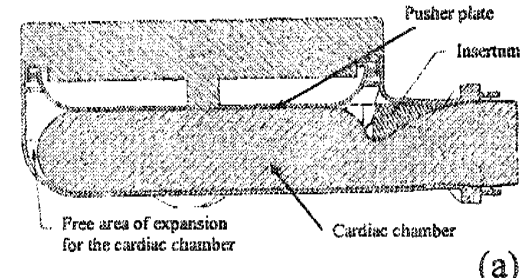

(a)

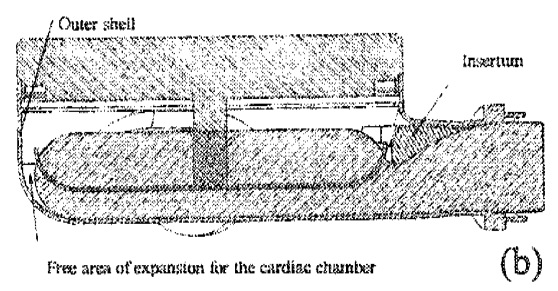

(b)

At the reverse motion, when the piston returns to its original position, there is flow in the inflow tube, while the outflow one is closed (filling phase). For the present study, considering a natural frequency of $100 \mathrm{bpm}$ (condition under stress), the cardiac cycle presents an ejection phase of $287 \mathrm{msec}$ and a filling phase of $313 \mathrm{msec}$.

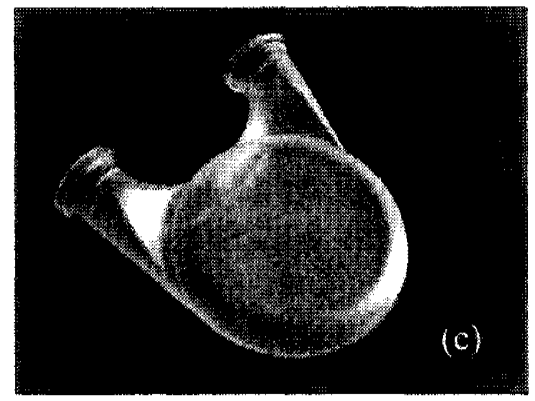

Figure 1: LVAD prototype cross sections (a. diastole phase; b. systole phase) and cardiac chamber membrane view (c) 


\section{Modeling}

\subsection{Theoretical model}

The CFD model implemented by means of FIDAP [1] allows for the resolution of the three-dimensional, time-dependent Navier-Stokes equations governing the conservation of mass and momentum:

$$
\begin{gathered}
u_{j, j}=0 \\
\rho\left(\frac{\partial u_{i}}{\partial t}+u_{j} u_{i, j}\right)=\sigma_{i j, j}+\rho f_{i}
\end{gathered}
$$

being $u_{i}$ and $\sigma_{i j}$ components of the velocity vector and the stress tensor respectively, while $f_{i}$ are the components of the body force per unit mass.

\subsection{Boundary conditions}

The numerical resolution of the equations of motion imposes the definition of appropriate boundary conditions for the fluid dynamic, structural and remeshing solvers, respectively. In particular zero fluid velocity condition is established at the inlet and outlet port to simulate the closure of the valve during the ejection and filling phase. The same condition on velocity is imposed at the wall surfaces. Zero displacements condition is attribuited to nodes in contact with the external rigid shell, as well as to inlet/outlet sections, while imposed displacements are set to all nodes of the moving membrane surface.

\subsection{Moving boundary}

During the filling phase when the pusher plate is not in contact with the membrane, motion and relative deformation of the clinical VAD cardiac chamber membrane are complex, tending to bend and buckle non-uniformly as a consequence of its low stiffness. Membrane structural behavior is then difficult to be captured when treated in fully coupled fluid-structure interaction and is cause of computational failure [2].

Therefore a simplified moving boundary has been applied such to produce the same general flow occurring in the clinical application. The moving boundary is such to describe the stroke volume variation through time by means of a pure function of the stroke length. All nodes that during the ejection phase come into contact with the piston are supposed to maintain their position without splippage. In the filling phase an aximmetric configuration of the deforming membrane is imposed. 


\subsection{Physical properties}

Blood is modeled as a Newtonian fluid with constant physical properties, being the assumption of linearity between the tangential stress and the velocity gradient sufficiently adequate when treating non confined flows. The deformable cardiac chamber membrane is assumed to behave as an hyperelastic material with structural behavior according to the Odgen law [3]:

$$
\sigma_{\gamma}=\lambda_{\gamma}^{2} S_{\gamma}=\sum_{r} \mu_{r}\left[\lambda_{\gamma}^{\alpha_{r}}-\left(\lambda_{1} \lambda_{2}\right)^{-\alpha_{r}}\right]
$$

being $\sigma_{\gamma}$ the true stress given by:

$$
\begin{aligned}
& \sigma_{\text {TRUE }}=\sigma_{E N G}\left(1+\varepsilon_{E N G}\right) \text { and } \varepsilon_{T R U E}=\ln \left(1+\varepsilon_{E N G}\right) \\
& \lambda_{\gamma}=1+\varepsilon_{T R U E_{\gamma}}
\end{aligned}
$$

and $\mu_{\mathrm{r}}$ the material constants. Material properties employed in the simulations are reported in Table 1 .

\section{Scenarios model}

\subsection{Scenarios configuration}

The complete cardiac cycle of ejection and filling is simulated. Two different sets of 3D analyses have been developed: a simple laminar coarse model and a refined turbulent model, the latter including the presence of the inlet valve, which is critical within the overall cycle.

Ejection phase: starting from the given motion field of the blood inside the cardiac chamber registered at the end of the filling phase, the movement of the elastic membrane under the rigid movement of the piston is simulated from the undeformed configuration to the maximum impressed displacement. The ejection phase starts at the time of piston-membrane contact in the real device: at that instant closure of the inlet valve and opening of the outlet valve are supposed to occur. The maximum displacement of the cardiac membrane $(17 \mathrm{~mm})$ equals the stroke length.

Filling phase: starting from the motion field established at the end of the ejection phase, the filling of the cardiac chamber is simulated with blood flowing through the inlet valve from the natural ventricle. The filling phase occurs when the piston moves from its maximum elongation to recover to the original position.

Effects induced by the inlet valve: at its entrance in the cardiac chamber, blood main flow and direction is influenced by the inlet valve position and relative aperture. The selected configuration considered a single spatial fixed position of the valve, in particular that one assumed by the valve for longer time throughout the cardiac cycle. 


\subsection{Meshing}

The numerical domain is subdivided into subdomains: inlet and outlet conduits (prolonged to avoid numerical instability due to ceased high velocity gradients) and cardiac chamber. The fluid domain is discretised by means of brick ( 8 nodes) elements whilst the membrane with shell elements, which do not support any stress state along the thickness. The coarse laminar model summed up to 35.000 elements, whilst the refined turbulent model is characterised by over 70.000 elements (Figure 2).

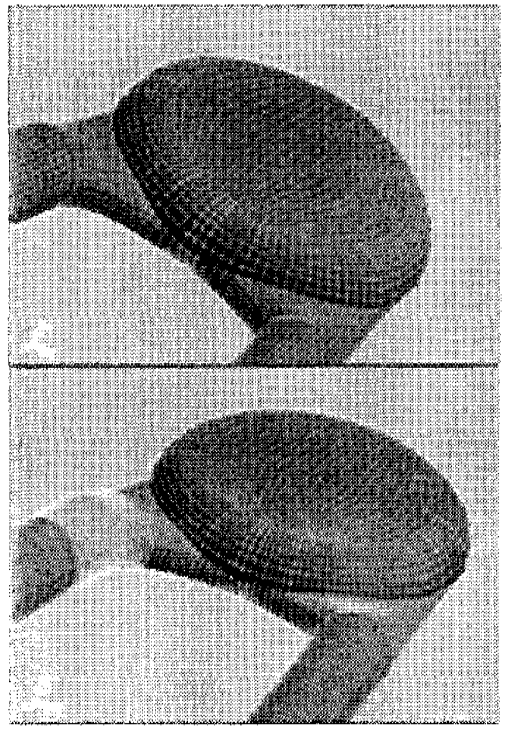

(a)

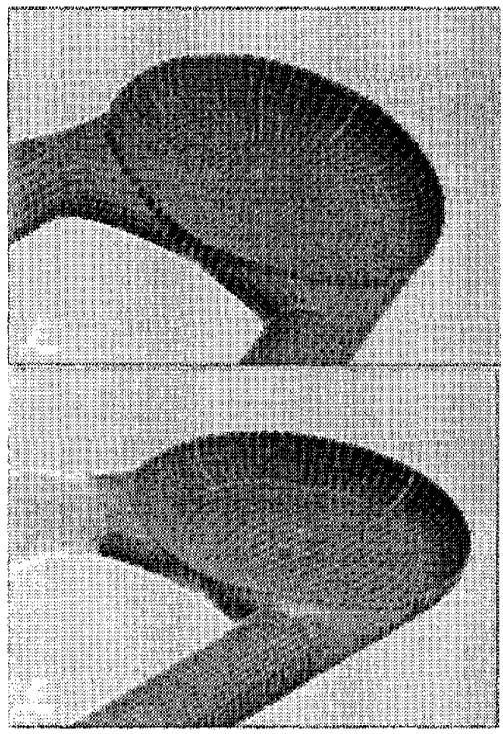

(b)

Figure 2: LVAD prototype membrane mesh, coarse (upper) and refined with inlet valve models (lower), undeformed (a) and deformed (b) views

\section{Numerical results}

Three different instants $(200,412$ and $537 \mathrm{msec})$ characteristic of the flow history within the medical device are selected to appropriately show the numerical results. These instants have direct correspondence with the deformation history of the cardiac membrane (Figure 3). Results from the coarse laminar model resulted to match very closely with the available experimental flow images and then are the sole presented in the following. Velocity plots reported refer to the mid horizontal plane of the blood volume.

Ejection: the flow field is dominated by the outflow and the penetration of the piston. As a result of the outflow, the entire fluid volume is directed to the outlet tube except for a small part which is trapped in the inlet area (Figure 4a). 
Points selected for numerical results presentation
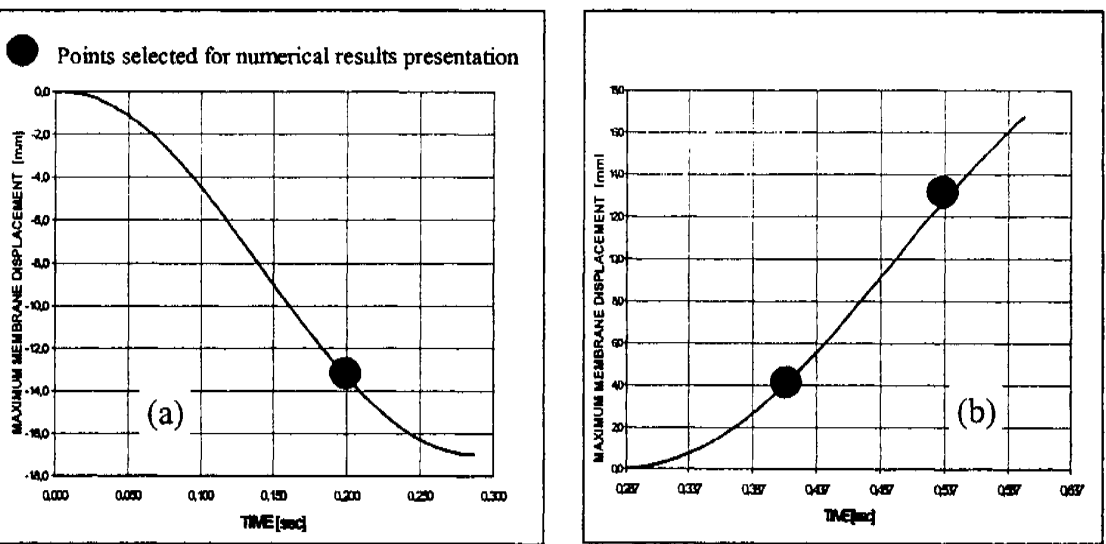

Figure 3: History of the membrane displacement during ejection (a) and filling (b)

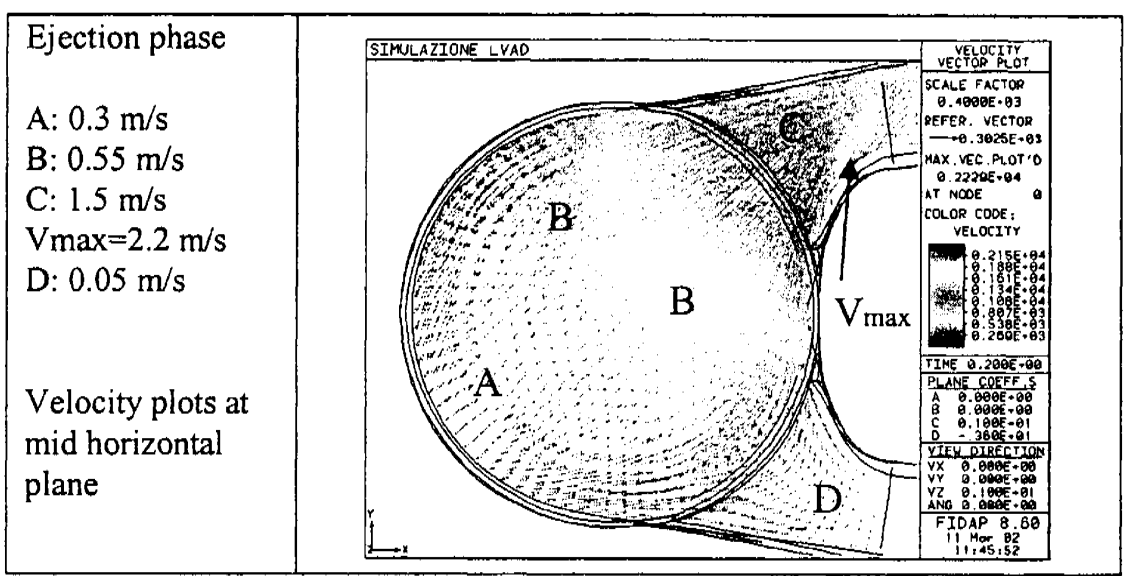

Figure 4a: Laminar model: velocity plot at $200 \mathrm{msec}$

Early filling: when the incoming jet enters the chamber a separation forms between the jet and the fluid attached to the wall because of the discontinuity at the junction of inlet tube and the chamber. A large vortex builds up during filling and causes the new and resident fluid to mix, but there is still a large section of unmixed, residual fluid in the right side of the chamber (Figure 4b).

Late filling: when the inflow jet is completely formed, the trapped fluid in the separation region starts to mix with the rest of the incoming jet, while a secondary vortex initiates in the mid area between the center of the chamber and the outlet section, thus allowing the mixing of the residing fluid (Figure 4c). 


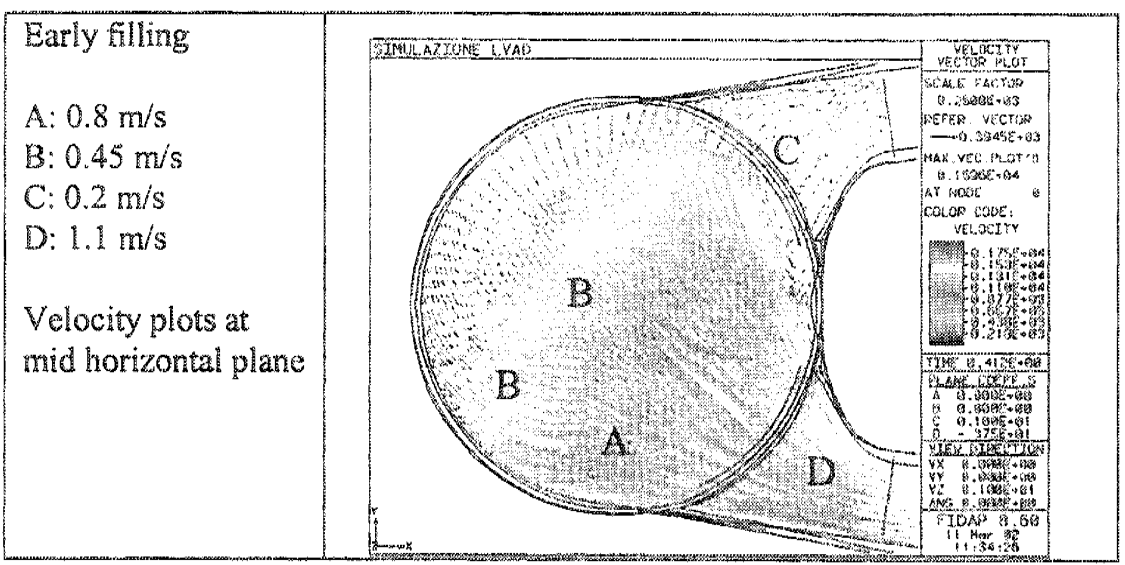

Figure 4b: Laminar model: velocity plot at $412 \mathrm{msec}$

\begin{tabular}{|l|l|l|l|l|l|}
\hline Late filling & A: $1.2 \mathrm{~m} / \mathrm{s}$ \\
$\mathrm{B}: 0.8 \mathrm{~m} / \mathrm{s}$ \\
$\mathrm{C}: 0.4 \mathrm{~m} / \mathrm{s}$ \\
$\mathrm{D}: 1 \mathrm{~m} / \mathrm{s}$ \\
Velocity plots at \\
mid horizontal \\
plane
\end{tabular}

Figure 4c: Laminar model: velocity plot at $537 \mathrm{msec}$

Maximum wall shear stress in the laminar analysis as well as the turbulent stress predicted by the turbulent model are below the threshold value identified for the blood cells rupture. This result confirms previous conclusions obtained by Jin and Clark [4] on sac-type ventricular assist devices.

\section{Experimental results}

Experimental results obtained during a previous test campaign by using flow visualisation techniques are used for comparison with numerical issues. Light scattering air bubbles of an average size of $300 \mu \mathrm{m}$ were employed to track fluid particle trajectory while recording was made by means of a special camera allowing superposition of multiple images with selectable exposure times.

Flow visualisation records at different phases are shown in Figure 5. 

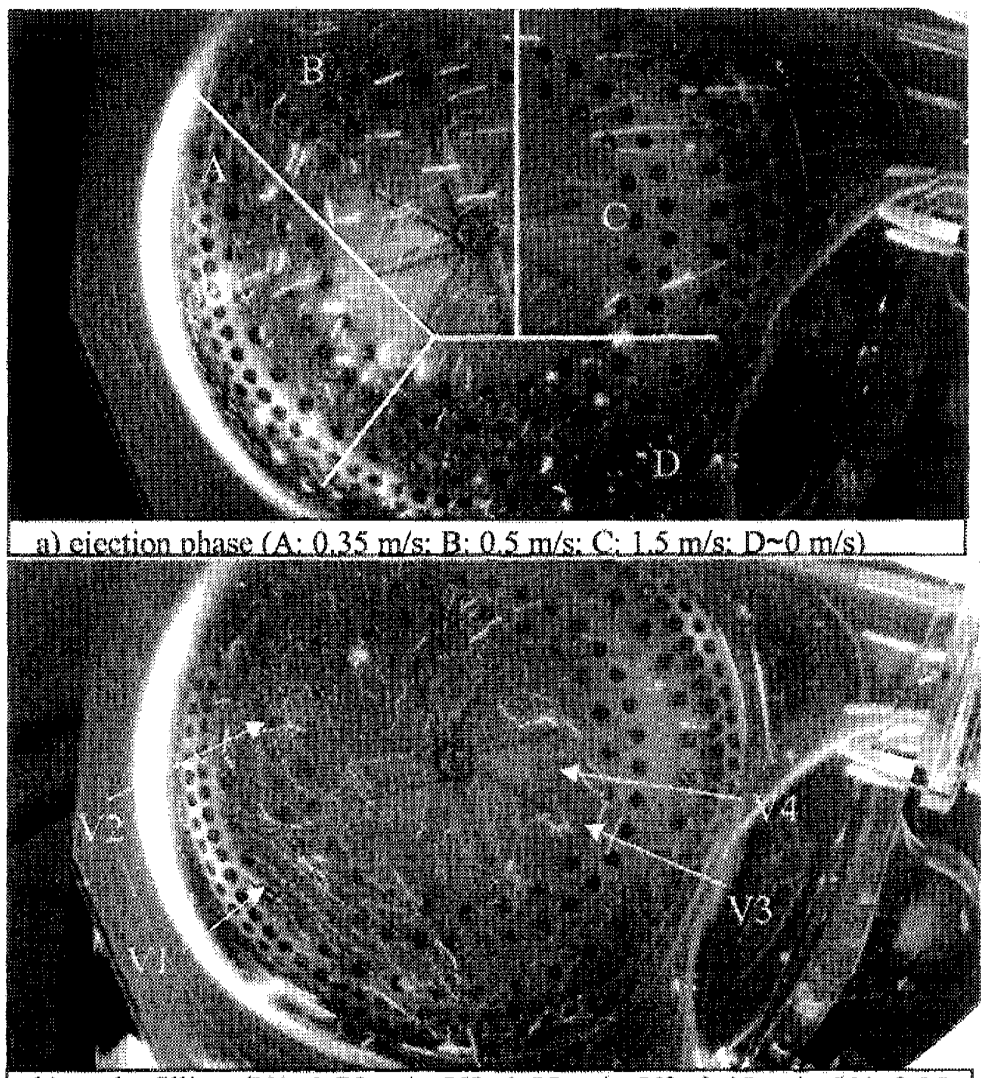

b) early filling (V1 $=0.75 \mathrm{~m} / \mathrm{s} ; \mathrm{V} 2=0.55 \mathrm{~m} / \mathrm{s} ; \mathrm{V} 3=0.45 \mathrm{~m} / \mathrm{s} ; \mathrm{V} 4=0.35$

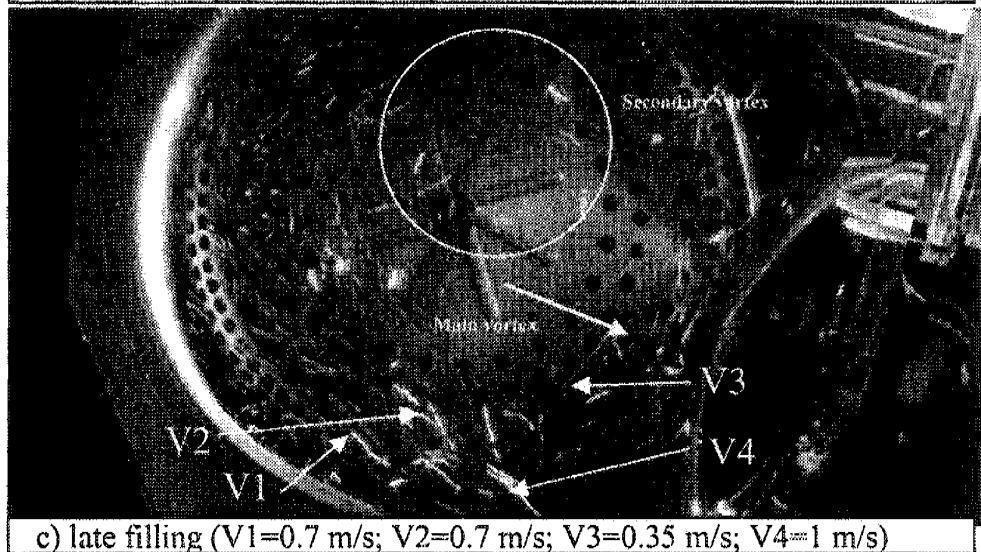

Figure 5: Flow visualisation records at different cardiac cycle phases 
Some quantitative patterns and measurements are indicated. The ejection phase is characterised by a sustained momentum of the flow in the center region, as revealed in the numerical simulation, while a backflow region is observed in the inlet area. Fluid velocity in the re-circulation area (A) is around $0.3-0.4 \mathrm{~m} / \mathrm{sec}$, whilst the outflow velocity reaches $1.5 \mathrm{~m} / \mathrm{sec}$. Then the jet penetrates the chamber fluid, which has been decelerated by the outlet valve closure, and induces retention of fluid at its left for a longer extent than that one predicted by simulation. Fast jet fluid particles velocity is $0.8 \mathrm{~m} / \mathrm{sec}$, but it decreases to less than $0.5 \mathrm{~m} / \mathrm{sec}$ in the nearby the conduits. At late filling, a second recirculation area with fluid velocity around $0.3 \mathrm{~m} / \mathrm{sec}$, seems effectively to add to the principal one, recalling the fluid resided at the rebound of the outlet valve closure. Measured fluid jet velocity $(1 \mathrm{~m} / \mathrm{sec})$ is slightly lower than that one numerically predicted $(1-1.2 \mathrm{~m} / \mathrm{sec})$.

\section{Conclusions}

Three-dimensional fluid dynamic analyses have been developed to investigate the characteristics of the flow motion in the complete cardiac cycle of a LVAD prototype. Predicted flow motion characteristics in the laminar regime were revealed to be in a fairly good agreement with experimental evidence. Vortices that develop in the chamber during filling are judged to increase blood mixing and provide favourable washing of the chamber wall.

The computational approach adopted has the potential to provide fast evaluation of new devices and to streamline design optimisation. More complex simulation scenarios including the physical presence of valves and the turbulence models can be adopted once the candidate device geometry has been preliminarily assessed.

Future studies will address non-axisymmetric deformation of the cardiac membrane to verify if adverse configurations can induce retention of fluid between the jet and the chamber wall for too many cardiac cycles, thus causing risk of blood cells stagnation.

\section{Acknowledgement}

This work was developed under the grant of the Italian Ministry of University and Scientific and Technological Research (Law 488/92) to the BIOPROSIM research project, leaded by ENEA.

The author wants to thank Dr. Carla Carabbio for precious support on medical aspects provided along the development of the work and his colleague Gian Maria Manfredini for suggestions during review of the paper. 


\section{References}

[1] Fluent Inc., FIDAP 8.6 - User's Manual, 2001.

[2] König, C. S., Clark, C., Mokhtarzadeh-Dhghan, M. R., Investigation of unsteady flow in a model of a ventricular assist device by numerical modelling and comparison with experiments, Medical Engineering \& Physics, 21, pp. 53-64, 1999.

[3] Gruttmann, F., Taylor, R. L., Theory and finite element formulation of rubberlike membrane shells using principal stretches, International Journal for Numerical Methods in Engineering, 35, pp. 1111-1126, 1992.

[4] Jin, W., Clark, C., Experimental investigation of unsteady flow behaviour within a sac-type ventricular assist device (VAD), Journal of Biomechanics, 26, pp. 697-707, 1993.

Table 1: Material property constants

\begin{tabular}{|c|c|}
\hline 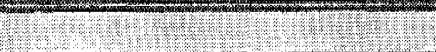 & M. \\
\hline Density & $1070 \mathrm{Kg} / \mathrm{m}^{3}$ \\
\hline Dynamic viscosity & $0.003 \mathrm{Pas}$ \\
\hline POIURE & MEMBR $A$ NE \\
\hline Young modulus & $20 \mathrm{MPa}$ \\
\hline Density & $1250 \mathrm{Kg} / \mathrm{m}^{3}$ \\
\hline $\begin{array}{l}\text { Constitutive law material } \\
\text { constants }\end{array}$ & $\begin{array}{l}\text { Parameters derived from non- } \\
\text { linear fitting of material data of } \\
\text { simple tension specimens testing. }\end{array}$ \\
\hline$\alpha_{1}=-1.28872 ; \alpha_{2}=-5.52885$ & $\mu_{1}=-11.8636 ; \mu_{2}=-1.03342$ \\
\hline$\alpha_{4}=-4.12686 ; \alpha_{3}=-2.56176$ & $\mu_{3}=-2.94132 ; \mu_{4}=-3.90366$ \\
\hline$\alpha_{5}=1.57622$ & $\mu_{5}=-15.9167$ \\
\hline
\end{tabular}

